\title{
Comparative Study of Sevoflurane and Isoflurane Anesthesia for the Long-term Safety Evaluation of Visual Prosthesis with Rabbits
}

\author{
Mariko Kuwabara, ${ }^{1 *}$ Hiroyuki Tashiro, ${ }^{2}$ Yukari Nakano, ${ }^{1,3}$ \\ Yasuo Terasawa, ${ }^{3}$ Hajime Sawai, ${ }^{4}$ and Jun Ohta ${ }^{1}$ \\ ${ }^{1}$ Graduate School of Materials Science, Nara Institute of Science \& Technology, \\ 8916-5 Takayama-cho, Ikoma, Nara 630-0192, Japan \\ ${ }^{2}$ Department of Health Sciences, Faculty of Medical Sciences, Kyushu University, \\ 3-1-1 Maidashi, Higashi-ku, Fukuoka 812-8582, Japan \\ ${ }^{3}$ Artificial Vision Institute, Research and Development Division, Nidek Co., Ltd., \\ 13-2 Hama-cho, Gamagori, Aichi 443-0036, Japan \\ ${ }^{4}$ Graduate School of Nursing, Osaka Prefecture University, \\ 3-7-30 Habikino, Habikino, Osaka 583-8555, Japan
}

(Received July 31, 2017; accepted October 17, 2017)

Keywords: sevoflurane, isoflurane, visual prosthesis, long-term safety evaluation, animal experiment

The development of a visual prosthesis requires long-term safety evaluation. Functional evaluation using evoked potential, such as visual evoked potential (VEP) and electrical evoked potential (EEP), is effective for this safety evaluation. We have experienced fatal cases, likely due to repeated isoflurane (IF) anesthesia, in chronic VEP recording. IF anesthesia may cause malignant hyperthermia, respiratory depression, and even death as a result of repetitive use. Sevoflurane (SF) is a safer anesthetic with a low blood/gas partition coefficient, and the introduction of and arousal from anesthesia can be performed quickly. Since there is no report on VEP in rabbits using SF anesthesia, we investigated whether SF anesthesia can be used as an alternative to IF anesthesia in evoked-potential measurement for the evaluation of the longterm safety of visual prostheses. The first negative wave $\left(\mathrm{N}_{1}\right)$ was used for the VEP evaluation of rabbits. In both anesthesia methods, obvious $\mathrm{N}_{1}$ peaks were obtained and the latencies were similar. Furthermore, the $\mathrm{N}_{1}$ amplitude under SF was higher than under IF. The difference in $\mathrm{N}_{1}$ amplitudes and latencies at different concentrations of each anesthetic was not statistically significant. Therefore, we found SF to be an alternative to IF in the VEP evaluation of visual prostheses in rabbits.

\section{Introduction}

A visual prosthesis is an implantable aid for people with acquired visual impairment. It can provide them with vision via electrical stimulation of their remaining visual systems based on data from a video camera. The retinal prosthesis, a type of visual prosthesis that stimulates the retinal circuitry of an impaired retina under conditions such as age-related macular degeneration and retinitis pigmentosa, has attracted attention, and the research and development of the *Corresponding author: e-mail: kuwabara.mariko.kd8@ms.naist.jp http://dx.doi.org/10.18494/SAM.2018.1715 
prosthesis has been active for these two decades. ${ }^{(1)}$ We have been developing a suprachoroidaltransretinal-stimulation retinal prosthesis using a 49-channel electrode for clinical trial. ${ }^{(2)}$

Prior to practical application in human subjects, animal experiments are necessary to prove that the prosthetic devices are functionally stable in vivo for long periods. Evoked potentials by retinal stimulation enable the evaluation of the transmission of visual information. Therefore, the safety assessment of a visual prosthesis can be achieved by measuring evoked potentials, such as electrical evoked potentials (EEPs), visual evoked potentials (VEPs), and electroretinogram (ERG), with implanted long-term stimulating electrodes and recording electrodes. The evaluation of EEPs reveals the long-term stability and efficiency of the stimulation electrode, whilst VEPs from full-field photic stimulation are indicative of the stability of the recording system (i.e., whether it is operating in a physiologically effective state with the chronic implanted recording electrode); ERG primarily confirms the maintenance of outer retinal functions. A combination of these electrophysiological recordings can tell us the cause of changes in stimulus response, allowing for safety evaluation. Additionally, according to the U.S. Food and Drug Administration (FDA) guidelines, the measurement of evoked potentials via long-term in vivo tests is required, if possible. ${ }^{(3)}$

Rabbits are used extensively for ophthalmological and visual neurophysiological studies, owing to both the similarity of the eyeball size compared with humans and excellent wound healing capabilities. ${ }^{(4)}$ In order to evaluate the long-term safety of visual prostheses using rabbits, we developed an evoked potential recording system ${ }^{(5)}$ and a chronic stimulation evaluation system. ${ }^{(6)}$ VEPs are affected by both the recording electrode and anesthesia. Thus, we developed novel recording electrodes with a smooth surface platinum (Pt) ball, $\mathrm{Pt}$ balltip electrode, and platinum/iridium (Pt/Ir) ball-tip planar multi-electrode array (MEA). We succeeded in long-term stable recording using these electrodes. ${ }^{(5)}$ Injected anesthetics, such as ketamine/xylazine or pentobarbital, were used in the previous study. ${ }^{(7)}$ These injected anesthetics caused difficulties in controlling the depth of anesthesia, and a high potential for death due to repeated anesthesia was noted. ${ }^{(8)}$ In addition, VEPs were only recorded during a short period just before awakening, since they disappeared in deep anesthesia and their amplitudes varied with the depth of anesthesia; hence, VEP measurement under fixed conditions is difficult. ${ }^{(9)}$ By contrast, when using isoflurane (IF) in inhalation anesthesia, VEP can be observed during deep anesthesia under fixed conditions, and anesthetic depth can be adjusted stably. Moreover, changes over time can also be observed. ${ }^{(5)}$ Nevertheless, deaths occurred occasionally owing to repeated IF anesthesia in our long-term VEP recording studies. Such accidental loss of animals disturbs not only our long-term tests but also the basic philosophy of animal experimentation, "The three Rs" (3Rs) advocated by Russell and Burch in 1959 (Replacement: replace the use of animals with alternative techniques; Reduction: reduce the number of animals used to a minimum; Refinement: refine the way experiments are carried out to ensure animals suffer as little as possible). ${ }^{(10)}$ Thus, such accidents should be minimized as much as possible. In animal experiments, IF, frequently used as inhalation anesthesia, may cause malignant hyperthermia and respiratory depression as a side effect, and even death due to repetitive anesthesia. ${ }^{(11,12)}$ By contrast, sevoflurane (SF), which is also used as inhalation anesthesia, is safer with a lower partition coefficient of blood/gas. Thus, the anesthetic 
transition is fast and introduction of/arousal from anesthesia can be performed quickly. ${ }^{(13-15)}$ $\mathrm{SF}$ anesthesia has been used for in vivo evoked potential measurements, but not yet in rabbits. ${ }^{(16,17)}$ There have been some studies of evoked potentials, such as VEP in IF anesthesia targeting rabbits; ${ }^{(18)}$ however, there are no reports on rabbit-targeted VEP and EEP studies using SF anesthesia.

The purpose of this study was to investigate whether SF anesthesia may be used as an alternative to IF anesthesia in evoked potential measurement for the assessment of the long-term safety evaluation of visual prostheses. We also examined the differences between IF and SF anesthesia, and the effects of anesthesia concentration on each VEP parameter.

\section{Materials and Methods}

\subsection{Implanted electrodes}

Three Japanese white rabbits (2.5-3.5 kg, male, Oriental BioService, Japan) were used in this study (identified as rabbit $\mathrm{A}$, rabbit $\mathrm{B}$, and rabbit $\mathrm{C}$ ). A recording electrode with a Pt ball (1.5 $\mathrm{mm}$ diameter) attached to the tip of a polyimide screw $\left(12.0 \mathrm{~mm}\right.$ long) was used [Fig. 1(a)]. ${ }^{(5)}$ The threaded insert, made of SUS303 (Ensat 302000025.500, kkv, Japan), was implanted $9 \mathrm{~mm}$ anteriorly and $6 \mathrm{~mm}$ right-laterally from the lambda suture, and the recording electrode was screwed into this threaded insert [Fig. 1(b)]. The implantation depth of the recording electrode was adjusted via the threaded insert while recording the VEP, and fixed when the amplitude of the VEP reached a maximum; ${ }^{(5)}$ the recording electrode was subsequently fixed with dental cement (UNIFAST TRAD, GC, Belgium). The reference electrode (M2 stainless-steel anchor screw; AM2-8, Unique Medical, Japan) was implanted $16 \mathrm{~mm}$ anteriorly to the lambda suture. We employed a recovery period of over 2 weeks prior to conducting the following experiment.

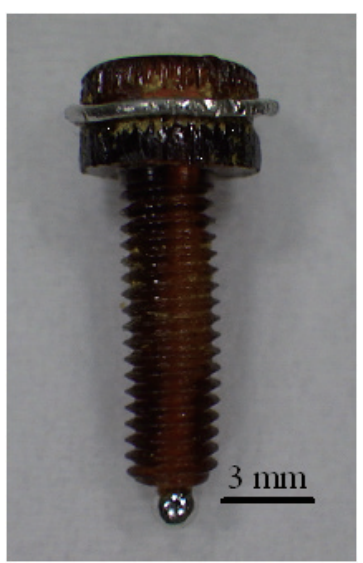

(a)

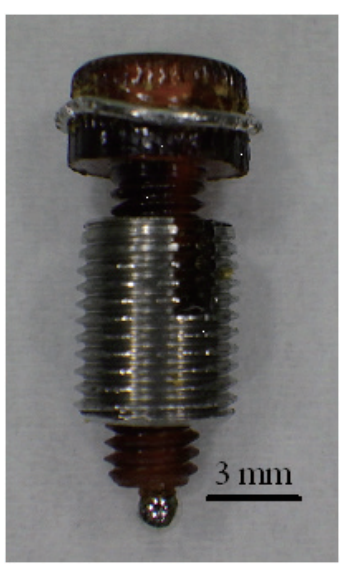

(b)

Fig. 1. (Color online) (a) Picture of the recording electrode. The recording electrode had the structure of a plastic screw with a platinum (Pt) ball at the tip. (b) Picture of the recording electrode with threaded insert. The implantation depth of the recording electrode was adjusted via the threaded insert to optimize the recording of VEPs. The Pt ball was placed in a subdural position. 


\subsection{Recordings of VEPs}

Each rabbit was allowed only water for $2 \mathrm{~h}$ prior to the experiments, after which it was placed in a recovery box with $4 \mathrm{~L} / \mathrm{min}$ of oxygen provided by anesthesia apparatus (NS-3000, Acoma, Japan). The room light was turned off, and the recovery box was covered with blackout curtains; the animal was adapted to the dark for $15 \mathrm{~min}$. Subsequently, vaporized anesthetic gas (IF: Forane, AbbVie, USA; SF: Sevofrane, AbbVie, USA) was introduced into the recovery box and monitored via a biological medical monitor (BP-608EV, Colin, Japan). Once sufficient anesthetic depth was achieved (the concentration in the recovery box was $2.4 \% \mathrm{IF}$, or $3.8 \% \mathrm{SF}$ ), the oxygen flow was reduced to $1 \mathrm{~L} / \mathrm{min}$ and the anesthetic was then delivered via a mask. A sufficient anesthetic depth was confirmed by absence of arousal response when the recovery box was shaken as an external stimulus. The pupil of the left eye (to be stimulated) was dilated with tropicamide (Mydrin-P, Santen, Japan), and additional local anesthesia was applied (oxybuprocaine hydrochloride; Benoxil, Santen, Japan). The right cornea was moisturized with topical application of hydroxyethyl cellulose (SCOPISOL, Senju, Japan), and then shaded from light with gauze. After perfect mydriasis, the eyelid was held open with an eyelid speculum and a contact lens electrode (EW-202, Mayo, Japan) was attached. For the body earth, a clip electrode (NE-103A, Nihon Kohden, Japan) was attached to the left ear with electroencephalogram paste. For maintaining body temperature, a circulating warm mat (TP 700, Stryker, USA) was used. VEPs were measured $15 \mathrm{~min}$ or more after the end-tidal anesthetic concentration reached the set condition under mask anesthesia (IF: 2.4, 2.0, and 1.5\%; SF: 4.0, 3.8, and 3.0\%). Measurement of VEPs under IF and under SF were carried out on different dates over two consecutive days. Light stimulation was performed via a contact lens electrode combined with a LED (LS-100, Mayo, Japan). The stimulus conditions were as follows, in accordance with standards of the International Society for Clinical Electrophysiology of Vision: luminance $3 \mathrm{~cd} \cdot \mathrm{s} / \mathrm{m}^{2}$ and duration $30 \mathrm{~ms}{ }^{(19)}$ The repetition frequency was set at $1 \mathrm{~Hz}$. The evoked potential was recorded with a bioamplifier (MEG-6116, AD-611J, Nihon Kohden, Japan) and data acquisition software (Eplyzer II, Kissei Comtec, Japan). Recording conditions were as follows: high-cut frequency (HCF) 100 $\mathrm{Hz}$, low-cut frequency (LCF) $1 \mathrm{~Hz}$, sampling frequency $10000 \mathrm{~Hz}$, and 32 count averages.

\subsection{Recordings of EEPs}

The left eyeball of the rabbit was fixed with nylon string threaded at the conjunctival limbus. The stimulation electrode was a Pt ball (diameter $2 \mathrm{~mm}$ ) attached to the tip of the glass electrode, and the return electrode (a $25 \mathrm{G}$ injection needle) was pierced into the superior limb. A manipulator (MMN-2, NARISHIGE, Japan) was used to modify the position of the stimulation electrode and search for the position where the EEP amplitude was the largest. Because EEP measurement with IF and SF were performed on different dates, the position where the stimulation electrode reaction was the strongest was marked with a polyester string; the next day, the mark was used as a guide for the stimulation electrode (Fig. 2). EEPs were measured $15 \mathrm{~min}$ or more after the end-tidal anesthetic concentration reached the set condition under mask anesthesia (IF: 2.4\%; SF: 3.8\%). The evoked potentials were recorded with an 


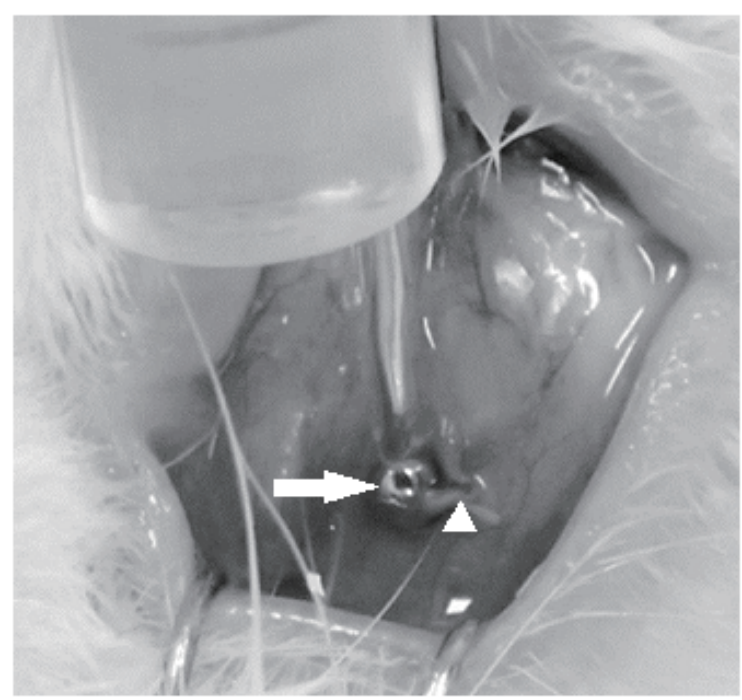

Fig. 2. Photoimage of the setup for electrical stimulation to the eye in order to record EEP. The platinum ball tip of the stimulating electrode (white arrow) was placed on the scleral surface of the elevated left eye ball. The symbol indicating the polyster string $(\Delta)$ was used as a guide for the stimulation position in EEP measurement.

induction potential research program (Eplyzer II, Kissei Comtec, Japan) and a bioamplifier (MEG-6116, AD-611J, Nihon Kohden, Japan). Electrical stimulation was performed with a cathodic first biphasic pulse, $1200 \mu \mathrm{A}$ current, $1 \mathrm{~ms}$ duration, and $2 \mathrm{~Hz}$ repetition frequency. Recording conditions were as follows: HCF $1000 \mathrm{~Hz}$, LCF $1.5 \mathrm{~Hz}$, sampling frequency 10000 $\mathrm{Hz}$, and 1000 count averages.

\subsection{Minimum alveolar concentration (MAC)}

One MAC is the concentration of a vapor in the lungs (measured as a percentage at 1 atmospheric pressure) needed to prevent movement (motor response) in response to pain stimulus in $50 \%$ of subjects. According to past studies, rabbits (New Zealand white rabbits) with 1 MAC IF anesthesia had a mean and standard deviation (SD) of $2.05 \pm 0.18 \%{ }^{(20)}$ whilst those with $1 \mathrm{MAC}$ SF anesthesia were $3.70 \pm 0.18 \%{ }^{(21)}$ In this study, IF MACs of $0.75 \mathrm{MAC}$, 1.0 MAC, and 1.2 MAC represented 1.5, 2.0, and 2.4\% end-tidal concentrations, respectively, and SF MACs of 0.79 MAC, 1.0 MAC, and 1.05 MAC represented 3.0, 3.8, and 4.0\% end-tidal concentrations, respectively.

\subsection{Analysis of VEPs}

Because the first negative component $\left(\mathrm{N}_{1}\right)$ of the VEPs was constantly recorded under IF and $\mathrm{SF}$ as described in the results, we measured the latencies and amplitudes of the $\mathrm{N}_{1}$ peaks by the peak-through method. ${ }^{(2)}$ Paired $t$-tests were used to estimate whether the $\mathrm{N}_{1}$ latencies or amplitudes differed between $1 \mathrm{MAC}$ IF and SF anesthesia. The significance level was set to $5 \%$. In addition, to determine whether there were significant differences between $\mathrm{N}_{1}$ latencies 
or $\mathrm{N}_{1}$ amplitudes depending on anesthetic concentrations, repeated measures analyses of variance (rANOVA) were performed. To evaluate the VEP waveforms, waveforms at 1 MAC (IF and SF) were compared. Statistical software (JMP Pro 13, SAS, USA) was used for the analysis.

All animal experiments were conducted in accordance with the Association for Research in the Vision Ophthalmology Statement for the Use of Animals in Ophthalmic and Visual Research, and institutional guidelines for the care and use of laboratory animals. Moreover, approval was obtained from the animal research committee at Nidek.

\section{Results}

The VEPs were consistently composed of a large negative component $\left(\mathrm{N}_{1}\right)$ followed by several small waves, as shown in Fig. 3. After $\mathrm{N}_{1}$, several small negative deflections were recorded, followed by a slow positive wave. As shown in Fig. 3, the VEP waveforms from three rabbits at 1 MAC represented a prominent first negative wave in both IF and SF anesthesia. In contrast, the small late waves after $\mathrm{N}_{1}$ varied in their peak amplitude and latency between the two anesthetics as well as between animals. The difference in $\mathrm{N}_{1}$ latencies between IF and SF was not statistically significant $(t=0.97, p=0.435$, Table 1$)$. However, $\mathrm{N}_{1}$ amplitudes of VEPs under SF anesthesia were significantly higher than those under IF anesthesia $(t=$ 5.18, $p=0.0353$, Table 1). Figure 4 shows the effects of different concentrations on VEP waveforms in IF and SF. Under both anesthetics, the $\mathrm{N}_{1}$ waves, indicated with black triangles, were recorded independently of their concentrations. Moreover, the $\mathrm{N}_{1}$ latencies seem to be

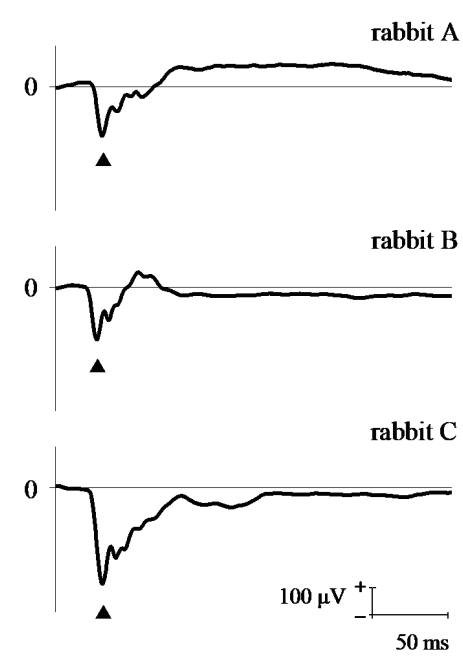

(a)

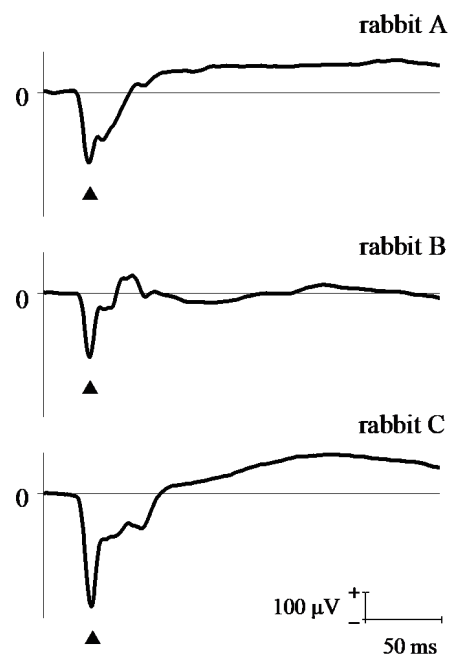

(b)

Fig. 3. VEP waveforms at 1 MAC. (a) VEP waveforms at an end-tidal concentration of $2.0 \%$ IF. (b) VEP waveforms at an end-tidal concentration of 3.8\% sevoflurane. $\boldsymbol{\Delta}$ represents the first negative peak $\left(\mathrm{N}_{1}\right)$. The amplitude of $\mathrm{N}_{1}$ was measured by the peak-through method. ${ }^{(22)}$ The latencies of $\mathrm{N}_{1}$ were measured from the time of starting flash stimulation to the time of the $\mathrm{N}_{1}$ peak. The flash stimulation started at $0 \mathrm{~s}$. 
Table 1

First negative peak $\left(\mathrm{N}_{1}\right)$ of VEP results under $1 \mathrm{MAC}$ anesthesia.

\begin{tabular}{lcc}
\hline Anesthesia & $\mathrm{N}_{1}$ latency $(\mathrm{ms})^{\mathrm{NS}}$ & $\mathrm{N}_{1}{\text { amplitude }(\mu \mathrm{V})^{*}}^{*}$ \\
\hline IF & $29.3 \pm 2.1$ & $239.6 \pm 89.4$ \\
SF & $30.4 \pm 0.9$ & $298.9 \pm 93.1$ \\
\hline
\end{tabular}

$n=3$. Values are mean $\pm \mathrm{SD}$.

${ }^{*}=$ significant $(p<0.05), \mathrm{NS}=$ not significant $(p>0.05$, paired $t$-test $)$.

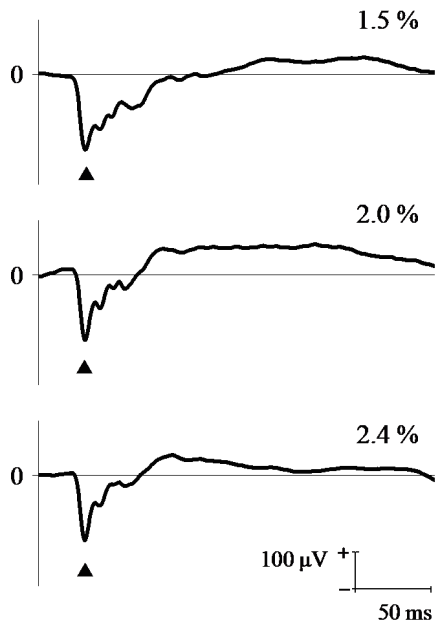

(a)

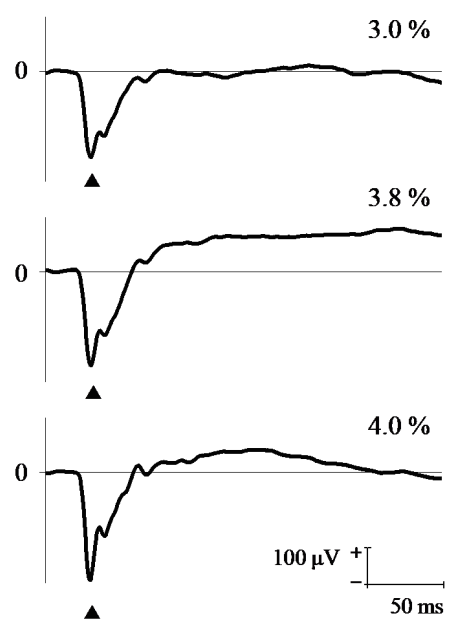

(b)

Fig. 4. VEP waveforms at different concentrations in rabbit A. (a) VEP waveform under IF anesthesia. (b) VEP waveform under SF anesthesia. $\Delta$ represents the first negative peak $\left(\mathrm{N}_{1}\right)$. The amplitude of $\mathrm{N}_{1}$ was measured by the peak-through method. ${ }^{(22)}$ The latencies of $\mathrm{N}_{1}$ were measured from the start time of flash stimulation to the time of the $\mathrm{N}_{1}$ peak. The flash stimulation started at $0 \mathrm{~s}$.

primarily unchanged. However, we observed latency reduction in some cases with the increase of anesthetic concentration, but the latency prolongation in others under IF [Fig. 5(a)]. In SF, the longest $\mathrm{N}_{1}$ latency was recorded at $1 \mathrm{MAC}$ for all three rabbits [Fig. 5(b)]. Overall, statistical analysis revealed no significant difference in $\mathrm{N}_{1}$ latencies for different anesthesia concentrations under IF or SF [rANOVA; IF: $F=0.29, p=0.7558$; SF: $F=1.57, p=0.2823$; Figs. 5(a) and 5(b)]. The differences in $\mathrm{N}_{1}$ amplitudes for different anesthesia concentrations under IF and SF were not statistically significant [rANOVA; IF: $F=0.21, p=0.8183$; SF: $F=0.30, p=0.7548$; Figs. 5 (c) and 5(d)]. As shown in Fig. 6, EEP could also be recorded under both IF and SF anesthesia.

\section{Discussion}

In the present study, we recorded the $\mathrm{N}_{1}$ component of the VEPs consistently under IF and $\mathrm{SF}$ anesthesia. $\mathrm{N}_{1}$ is presumed to be the first excitation in layer IV of the primary visual cortex elicited by geniculocortical inputs. ${ }^{(23)}$ On the other hand, the waves following $\mathrm{N}_{1}$ are thought to be secondary and later cortical responses to the subcortical afferents after layer IV, and the 


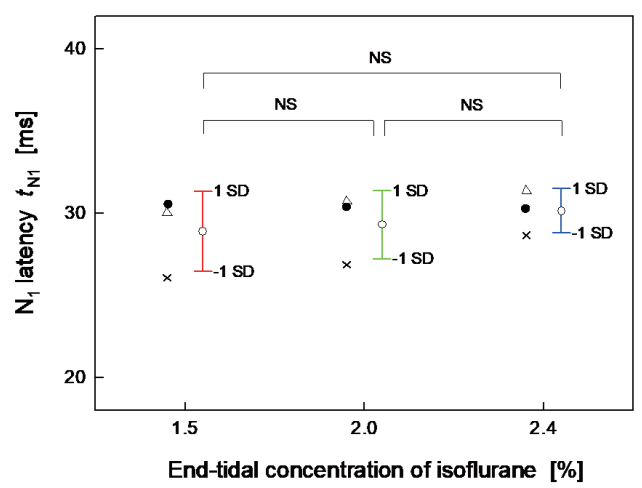

(a)

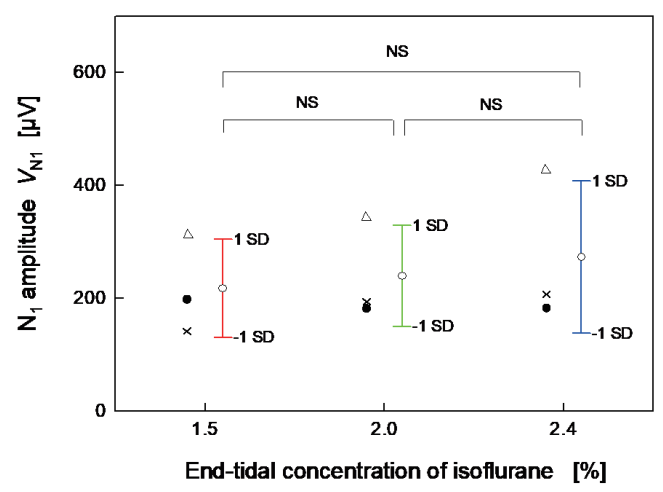

(c)

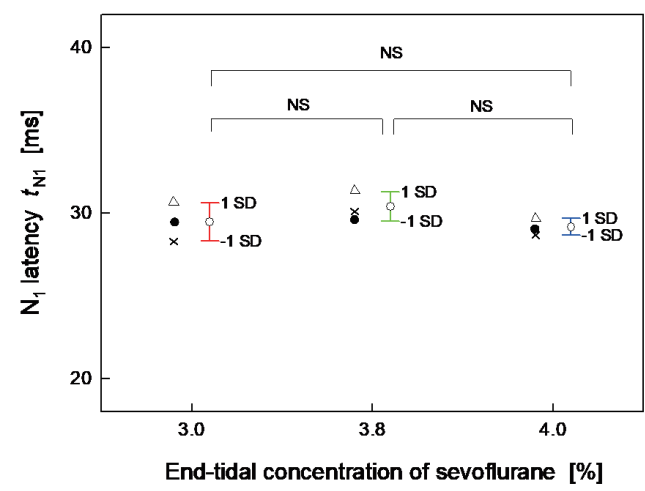

(b)

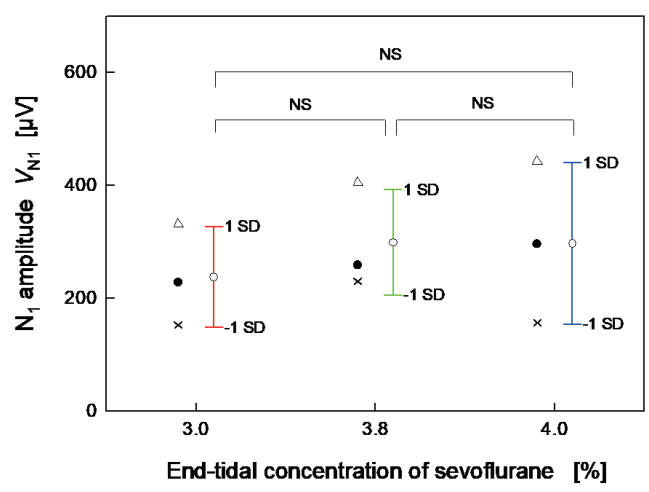

(d)

Fig. 5. (Color online) (a) First negative peak $\left(\mathrm{N}_{1}\right)$ latencies at different concentrations of IF. (b) $\mathrm{N}_{1}$ latencies at different concentrations of SF. (c) $\mathrm{N}_{1}$ amplitudes at different concentrations of IF. (d) $\mathrm{N}_{1}$ amplitudes at different concentrations of SF. The symbols are $\bullet, \times$, and $\Delta$ for rabbits A, B, and C, respectively. The white circles are the mean values, and the bars represent the range of $\pm 1 \mathrm{SD}$. [NS $=$ not significant $(p>0.05$; repeated measures analysis of variance)].

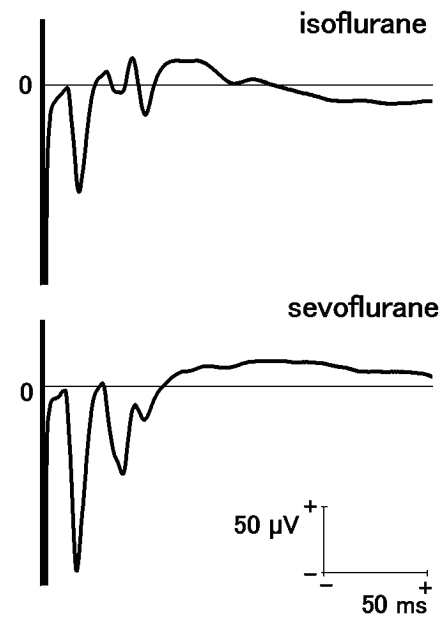

Fig. 6. EEP waveform in rabbit C. EEP stimulation was performed with a cathodic first biphasic pulse, $1200 \mu \mathrm{A}$ current, $1 \mathrm{~ms}$ duration, and $2 \mathrm{~Hz}$ repetition frequency. The electrical stimulation started at $0 \mathrm{~s}$. 
result of complicated reactions in the visual cortex with large variations and a lack of stability. ${ }^{(24)}$ Thus $\mathrm{N}_{1}$ can be a useful analytical target for reliably evaluating VEPs. In fact, in a previous study in which IF was used, the $\mathrm{N}_{1}$ of VEPs was analyzed. ${ }^{(25)}$ Thus, our successful recording of $\mathrm{N}_{1}$ confirms that $\mathrm{SF}$ is a good alternative to IF for long-term VEP evaluation in rabbits.

It is surprising that the peak latencies and amplitudes of $\mathrm{N}_{1}$ were stable and unaffected by changes in anesthesia concentration. In rats anesthetized with SF, it was demonstrated that the VEP latency may increase as SF concentration increases. ${ }^{(26)}$ The discrepancy between this report and our present study may be due to animal species, although the details are unknown and other possibilities remain.

We also found that the peak amplitudes of $\mathrm{N}_{1}$ under $\mathrm{SF}$ anesthesia were higher than those under IF anesthesia, despite the same concentration (1 MAC). The reason is considered to be that the effect of anesthesia on the visual nerve system and the visual cortex differs between IF and SF. For anesthesia management in animal testing, 1.2 to $1.5 \mathrm{MAC}$ is generally used. In our experience, the respiratory rate of the rabbits dropped at over 1.05 MAC in SF anesthesia, while this phenomenon did not occur at 1.2 MAC in IF anesthesia. $1 \mathrm{MAC}$ is the concentration of a vapor in the lungs that is needed to prevent movement (motor response) in response to pain stimulus in $50 \%$ of subjects. Therefore, the MAC value is not a value expressing the degree of effect for anesthesia on the visual nerve system and the visual cortex. Furthermore, according to the definition of the MAC, the depth of anesthesia other than 1 MAC differs depending on the anesthetic. The underlying mechanism of higher $\mathrm{N}_{1}$ amplitudes under SF remains to be elucidated.

In this study, SF anesthesia allowed us to record EEPs as well as VEPs. EEP measurements under SF and IF anesthesia were explored on different days, referring to polyester thread markers, but it was difficult to ensure that marker placement was achieved with perfect accuracy. EEP latencies vary depending on which cell responds in the retina, and the magnitude of the EEP amplitude is affected by the positional relationship between the stimulation electrode in the retina and the recording electrode in the cortex. ${ }^{(27)}$ Thus, in this study, we only explored whether EEP could be recorded or not. Comparison of latencies and amplitudes of EEP is difficult without implanted electrodes fixed in place. However, since VEP is a full-field stimulus, conditions can be the same even on different days, and the resulting parameters can be compared.

Regarding the anesthesia, studies on using repeated SF anesthesia with rabbits and mice have been conducted ${ }^{(28,29)}$ and there was no report of death due to repeated SF anesthesia. Thus, repeated SF anesthesia is safer than repeated IF anesthesia. Regarding long-term recording of the evoked potential, recording electrodes used in this study were developed and successful in stable evoked potential recording for 6 months under IF anesthesia was accomplished. ${ }^{(5)}$ In this study, it was revealed that SF can be an alternative to IF for VEP evaluation in rabbit. Therefore, long-term safety evaluation of visual prosthesis with the evoked potential can be performed more safely by using repeated SF anesthesia and a recording electrode capable of long-term evoked potential recording.

For the safety evaluation of visual prosthesis in long-term animal testing, FDA guidelines recommend implantation of the retinal prosthesis for at least 6 months, nevertheless, chronic 
stimulation for 6 months and functional evaluation over time are not required. ${ }^{(3)}$ However, it is considered that chronic stimulation and functional evaluation over time are also important. For that reason, the long-term safety evaluation for a visual prosthesis with functional devices was reported by Nayagam et al. ${ }^{(30)}$ We plan to perform 6-month chronic stimulation and assessments over time for the safety evaluation of our visual prosthesis by suprachoroidal transretinal stimulation..$^{(2,6)}$ The assessments include VEP, EEP, ERG, funduscopy, optical coherence tomography, measurement of ocular pressure, and histopathological evaluation. The chronic stimulation is started two weeks after the surgery, and the evaluation of this longterm safety will be performed under SF anesthesia in the following schedule: once every week from the beginning of the chronic stimulation until one month, once every two weeks from one month until three months, and once every month from three months until six months. After the stimulation period, the histopathological evaluation will be performed.

\section{Conclusions}

VEP under SF anesthesia enabled the observation of $\mathrm{N}_{1}$ with similar latencies to IF anesthesia. $\mathrm{N}_{1}$ amplitudes under $\mathrm{SF}$ anesthesia were equal to or higher than those under IF anesthesia. Thus, VEP under SF anesthesia could be evaluated by the same method as VEP under IF anesthesia. Furthermore, under SF anesthesia, the EEP waveform was similar to that under IF anesthesia. These results indicated that the physiological effects of IF and SF are similar, and that there are essentially no differences between the anesthesia methods for evaluating the function of the visual nervous system from retina to cortex. Under SF anesthesia, anesthetic concentrations can be maintained constantly, therefore, VEP evaluation under SF anesthesia is also possible even if the experiment is carried out on different days. We concluded that SF could be an alternative to IF for long-term evoked potential recording of functional maintenance and for the safety evaluation of visual prostheses. SF is a substitute for IF. Although in long-term animal testing, anesthesia causes changes in the amplitude of evoked potential, it is necessary to conduct long-term tests using the same anesthesia throughout the testing period. Measuring the time-dependent change in evoked potential using SF inhalation anesthesia is safer for animals and thus in accord with "The 3Rs" of animal experiments. Moreover, it would prevent dropout during long-term in vivo tests.

We have established a recording system capable of performing evoked potential recording safely, for longer periods of time, by combining the developed recording electrode ${ }^{(5)}$ with SF anesthesia. In the future, we will use SF anesthesia to evaluate the long-term safety of visual prostheses in vivo by a complex evaluation of EEP, VEP, and ERG.

\section{Acknowledgments}

The authors thank Hiroyuki Kanda and Takashi Fujikado of Osaka University for their expertise with the animal experiments and evaluation. 


\section{References}

1 L. Yue, J. D. Weiland, B. Roska, and M. S. Humayun: Prog. Retin. Eye Res. 53 (2016) 21.

2 T. Fujikado, M. Kamei, H. Sakaguchi, H. Kanda, T. Endo, M. Hirota, T. Morimoto, K. Nishida, H. Kishima, Y. Terasawa, K. Oosawa, M. Ozawa, and K. Nishida: Invest. Ophthalmol. Visual. Sci. 57 (2016) 6147.

3 Center for Devices and Radiological Health, Investigational Device Exemption (IDE) Guidance for Retinal Prostheses. U. S. Department of Health and Human Services, Food and Drug Administration (Silver Spring, 2013).

4 M. L. Khraiche, S. E. Emam, A. Akinin, G. Cauwenberghs, W. Freeman, and G. A. Silva: 2013 Conf. Proc. IEEE Eng. Medicine Biology Society (IEEE, 2013) 3539.

5 M. Kuwabara, H. Tashiro, Y. Terasawa, K. Osawa, T. Tokuda, J. Ohta, and T. Fujikado: Adv. Biomed. Eng. 6 (2017) 59.

6 H. Tashiro, Y. Terasawa, M. Kuwabara, K. Osawa, T. Tokuda, J. Ohta, and T. Fujikado: Adv. Biomed. Eng. 6 (2017) 8.

7 K. Nakauchi, T. Fujikado, H. Kanda, T. Morimoto, J. S. Choi, Y. Ikuno, H. Sakaguchi, M. Kamei, M. Ohji, T. Yagi, S. Nishimura, H. Sawai, Y. Fukuda, and Y. Tano: Graefes. Arch. Clin. Exp. Opthalmol. 243 (2005) 169.

8 W. R. Woodward, D. Choi, J. Grose, B. Malmin, S. Hurst, J. Pang, R. G. Weleber, and D. M. Pillers: Doc. Ophtalmol. 115 (2007) 187.

9 G. H. Rose, S. P. Gruenau, and J. W. Spencer: Electroencephalogr. Clin. Neurophysiol. 33 (1972) 141.

10 W. M. S. Russell and R. L. Burch: The Principles of Humane Experimental Technique (Methuen, London, 1959) Chaps. 5-7.

11 J. G. Wade and W. C. Stevens: Anesth. Analge 60 (1981) 666.

12 H. R. Kusuma, N. K. Venkataramana, S. A. Rao, A. L. Naik, D. Gangadhara, and K. H. Venkatesh: Indian J. Anaesth. 55 (2011) 290.

13 T. Kazama and K. Ikeda: Anesthesiology 68 (1988) 435.

14 E. I. Eger II and B. H. Johnson: Anesth. Analge. 66 (1987) 977.

15 A. Bergadano, R. Lauber, A. Zbinden, U. Schatzmann, and Y. Moens: Br. J. Anaesth. 91 (2003) 276.

16 M. Osawa, K. Shingu, M. Murakawa, T. Adachi, J. Kurata, N. Seo, T. Murayama, S. Nakao, and K. Mori: Anesth. Analge. 79 (1994) 52.

17 J. C. Murrell, H. N. de Groot, E. Psatha, and L. J. Hellebrekers: Am. J. Vet. Res. 66 (2005) 1156.

18 S. R. Montezuma, J. F. Rizzo III, and O. R. Ziv: J. Rehabil. Res. Dev. 41 (2004) 113.

19 J. V. Odom, M. Bach, M. Brigell, G. E. Holder, D. L. McCulloch, A. Mizota, and A. P. Tormene: Doc. Opthalmol. 133 (2016) 1.

20 J. C. Drummond: Anesthesiology. 62 (1985) 336.

21 M. S. Scheller, L. J. Saidman, and B. L. Partridge: Can. J. Anaesth. 35 (1988) 153.

22 H. A. Shah, S. R. Montezuma, and J. F. Rizzo III: Exp. Eye Res. 83 (2006) 247.

23 L. B. Padnick and R. A. Linsenmeier: Vision Res. 39 (1999) 2833.

24 C. E. Schroeder, C. E. Tenke, S. J. Givre, J. C. Arezzo, and H. G. Vaughan Jr: Vision Res. 31 (1991) 1143.

25 T. Okuno, H. Oku, and T. Ikeda: Neuro-Ophthalmology 26 (2002) 59.

26 A. M. Ghita, D. Parvu, R. Sava, L. Georgescu, and L. Zagrean: J. Med. Life 15 (2013) 214.

27 K. Wang, X.-Q. Li, X.-X. Li, W.-H. Pei, H.-D. Chen, and J.-Q. Dong: Vision Res. 51 (2011) 1897.

28 C. Kaymak, E. Kadioqlu, H. Basar, and S. Sardas: Hum. Exp. Toxicol. 23 (2004) 413.

29 A. Kilicaslan, M. Belviranli, N. Okudan, and A. E. Nurullahoqlu: Fundam. Clin. Pharmacol. 27 (2013) 641.

30 D. A. X. Nayagam, R. A. Williams, P. J. Allen, M. N. Shivdasani, C. D. Luu, C. M. Salinas-LaRosa, S. Finch, L. N. Ayton, A. L. Saunders, M. McPhedran, C. McGowan, J. Villalobos, J. B. Fallon, A. K. Wise, J. Yeoh, J. Xu, H. Feng, R. Millard, M. McWade, P. C. Thien, C. E. Williams, and R. K. Shepherd: PLoS One 9 (2014) 1. 